\title{
Una metodología de análisis del espacio doméstico polivalente
}

\author{
A methodology of analysis of domestic polyvalent spaces \\ Lucía Martín López* \\ Grupo de Investigación e Innovación en Educación. Desarrollo Territorial Sostenible. Escuela de Arte, \\ Arquitectura y Diseño. Tecnologico de Monterrey, Querétaro, México. \\ luciamartinlopez@gmail.com \\ María Fernanda Alcalá Meade** \\ Escuela de Arquitectura. Universidad Anáhuac México. México. \\ mafer_alcm@hotmail.com
}

* Doctora en arquitectura con mención internacional cum laude (2016) y Premio extraordinario de Doctorado 2015-16 por la Universidad Politécnica de Madrid (NPM). Coordinadora del Centro de Investigación en Arquitectura de la Universidad Anáhuac México, candidata del Sistema Nacional de Investigadores de CONACYT. Con su práctica profesional centrada en la vivienda, ha obteniendo numerosos premios en concursos internacionales y expuesto su trabajo en la EXPO Milano 2015, y en el PabeIlón virtual de España de la $16^{a}$ Bienal de Arquitectura de Venecia, entre otros.

** Diseñadora Industrial y estudiante de la Maestría de Arquitectura y Diseño de Interiores de la Universidad Anáhuac México 


\section{Resumen}

Un espacio polivalente es aquel capaz de albergar diversas aplicaciones de uso inciertas. Se considera que con un adecuado diseño de los espacios de una vivienda y de su articulación a través de la estructura y el mobiliario se puede potenciar la polivalencia de esta. Este artículo propone una metodología de análisis para espacios domésticos que mediante la recopilación de las teorías de John Habraken, Kiyoyuki Nishihara, Bernard Leupen y Alexander Klein es capaz de identificar estrategias de diseño para elaborar un espacio óptimo en términos de polivalencia.

Palabras claves: vivienda, representación gráfica, flexibilidad, polivalencia, mobiliario

\section{Abstract}

A polyvalent space host diverse applications of uncertain use. Polyvalence in a house can be enhanced with an adequate design of the spaces and its articulation through the structure and the furniture. This article proposes a methodology of analysis for domestic spaces that compilate theories of John Habraken, Kiyoyuki Nishihara, Bernard Leupen and Alexander Klein to identify design strategies to elaborate an optimal space in terms of versatility.

Keywords: housing, graphic representation, flexibility, versatility, furniture. 


\section{Sobre la importancia del espacio doméstico polivalente.}

El artículo cuarto de la Constitución Mexicana indica que "cada familia tiene el derecho a disfrutar una vivienda decente y decorosa", lo que significa que esta debe ser adecuada y cumplir ciertos criterios de asequibilidad, habitabilidad, accesibilidad, adecuación cultural, ubicación, disponibilidad de servicios, instalaciones e infraestructura.

Según el documento "Las dimensiones mínimas de vivienda de acuerdo a disposiciones y reglamentos oficiales" del INFONAVIT, la superficie útil de la vivienda mínima en Ciudad de México está comprendida entre 62 y 65 m2 y está destinada a familias de una a cuatro personas. Cabe destacar, sin embargo, que estos mínimos no se respetan ya que de acuerdo al Censo 2015 llevado a cabo por el Instituto Nacional de Estadística y Geografía (INEGI), sólo el $42.35 \%$ de la población vive en propiedades con un área mayor a los $60 \mathrm{~m} 2$, mientras que el $12.66 \%$ vive en un área menor a los 45 m2. (INFONAVIT, 2018)

Ante esta situación se plantea la cuestión de si es posible solucionar el déficit de vivienda desde un enfoque más cercano a la realidad mediante viviendas con superficies menores a los 65 m2. Dado que es complejo diseñar con precisión un espacio que se acomode a los requerimientos de toda una población, muchas de las iniciativas constructivas que destacan en la actualidad apuntan a hacer de la variabilidad y la adaptabilidad características importantes en todo proyecto. El presente artículo trata de encontrar las soluciones apropiadas para distribuir las actividades que se lleven a cabo dentro de estas viviendas mínimas adaptables a través de una metodología de análisis que permita evaluar el esquema de actividades y el equipamiento de estas.

Se parte, para ello, del concepto de polivalencia acuñado en 1991 en un debate arquitectónico por Herman Hertzberger, quien señalaba que la solución a este tipo de interrogantes estaba en conseguir que los espacios se utilizaran para más de una actividad de manera que se aprovechara al máximo cada metro cuadrado del espacio doméstico, (Leupen, 2006), entendiéndolo como "el espacio físico de las viviendas y sus modos de habitarlas". (Sánchez, 2004. P. 5). Se entiende por tanto la polivalencia como la posibilidad de cambio y mutabilidad de la vivienda de manera que la casa pueda proporcionar distintas soluciones para diferentes usos, sin alterar la estructura que la compone. (Leupen, 2006).

Según Carboni, la teoría de Hertzberger se desarrolla sobre la idea de entender la arquitectura como una obra al servicio de las personas capaz de responder de forma eficaz a sus necesidades materiales y psicológicas. Por consiguiente, el arquitecto tiene que ser capaz de diseñar un espacio que pueda ofrecer al usuario la oportunidad de elegir como modificar el uso de sus espacios según sus necesidades y entre distintas posibilidades. (Carboni, 2015). La polivalencia crea la capacidad en el espacio de generar soluciones pertinentes para cualquier situación que se pudiera generar. Por consiguiente, no es más que la capacidad de un espacio de albergar diversas aplicaciones inciertas. (Hertzberger, 2016).

La polivalencia dentro del ámbito arquitectónico implica, por tanto, el hecho de que un espacio pueda ser utilizado de distintas maneras sin alteraciones estructurales, atendiendo a la capacidad de intercambiar actividades en un mismo lugar.

\section{Elaboración de la metodología de análisis.}

A la hora de construir la metodología propuesta se hizo énfasis en el dibujo como herramienta de análisis comparativo entre diversos casos de estudio. Para ello, se estudiaron las teorías de varios arquitectos y se extrajeron sus sistemas de análisis gráfico para aplicarlos a esta metodología de manera que se complementaran las reflexiones de todos: de John Habraken se analizó la representación de la zonificación del espacio dentro de una 
vivienda; de Kiyoyuki Nishihara, las actividades principales que se llevan a cabo en una vivienda; de Bernard Leupen su forma de esquematización de las actividades de la vivienda; y de Alexander Klein la forma de expresar gráficamente el uso de superficies útiles versus libres, los esquemas en relación al espacio - actividad, la iluminación y la proyección de sombras, los recorridos principales dentro de la vivienda y la zonificación de actividades.

Esta información se sintetizó y calificó posteriormente en una tabla de análisis que permite evaluar un proyecto de vivienda mínima polivalente tal y como se verá en el apartado "Descripción del método de análisis” de este texto.

\section{La zonificación de espacios según John Ha- braken.}

En su libro "El Diseño de Soportes", John Habraken desarrolla un sistema en el cual una vivienda, construida o por construir, puede ser zonificada de modo que futuros ocupantes de esta puedan redistribuir los espacios.

Según Habraken, se pueden identificar cuatro razones por las que una persona desea modificar su propio entorno: Como medio de autoexpresión, por influencia de los cambios y estilos en la sociedad, por la introducción de avances tecnológicos o por encontrarse en alguna fase del desarrollo de una familia, lo cual no sólo implica un aumento/disminución en el número de habitantes, sino también un cambio en las actividades que se llevan a cabo dentro de un mismo espacio.

Para poder modificar o personalizar un espacio, hay que entender primero de que se compone este, para lo cual Habraken utiliza los términos soporte y unidad separable, indicando que un área puede ser diferenciada en dos partes: sobre la que el individuo tiene control, y sobre la que la comunidad decide colectivamente. (Habraken, 1979).

Entendemos por esto, que un soporte no es meramente el propio armazón estructural de una vivienda, sino que es una estructura diseñada y construida en un lugar específico y que no puede ser modificada. A su vez, las unidades separables no son los componentes que rellenan el espacio restante, si no cualquier elemento adaptable y capaz de ser usado en muchas combinaciones diferentes. "En el diseño de un soporte, el objetivo es encontrar una solución que permita todas las variaciones deseadas y que a la vez use las mínimas unidades separables posibles." (Habraken, 1979). De modo que el soporte idóneo es aquel que

Figura 01: Esquemas de zonificación de J. Habraken.
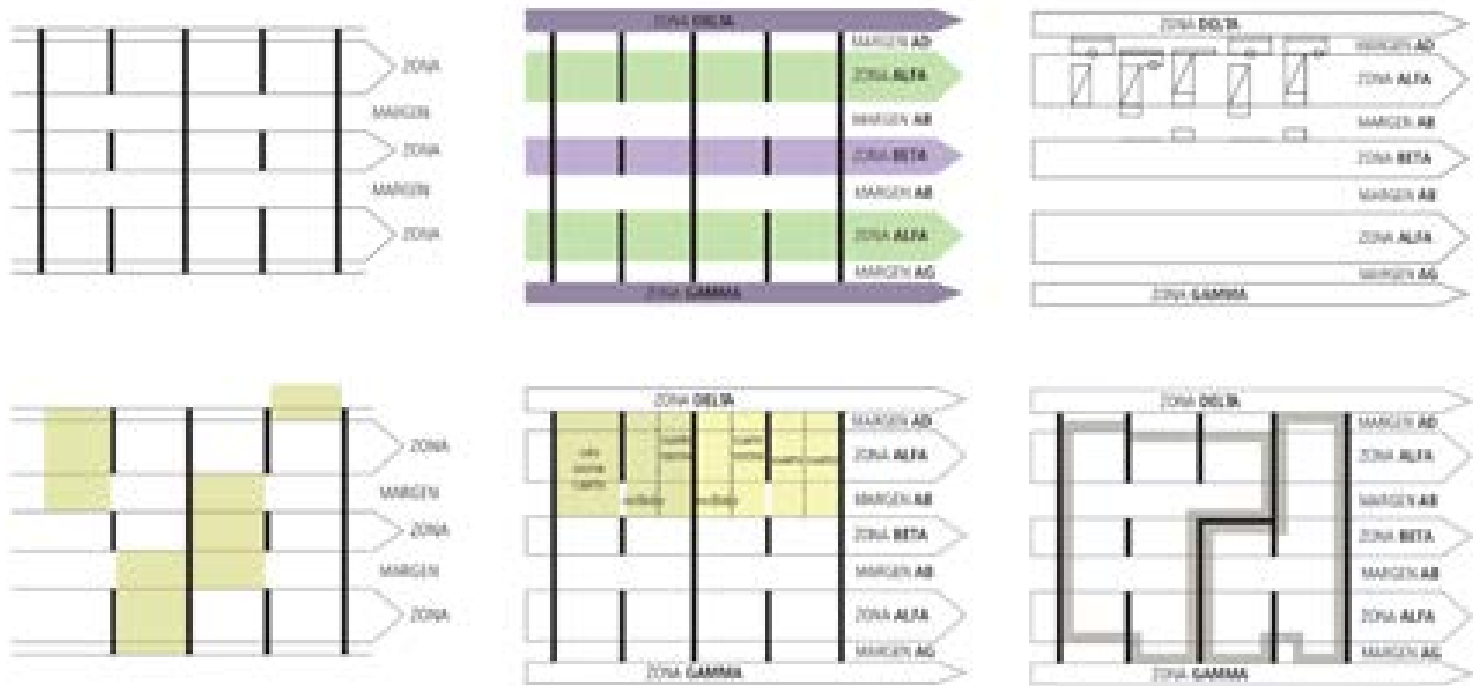

Fuente: Elaboración propia 
ofrece espacios específicos, que pueden ser reconocidos y que evoquen diversas posibilidades para permitir futuras adaptaciones.

La zonificación que nos ofrece el autor puede ayudarnos a identificar el modo en que se pueden ordenar o incluso agrupar áreas específicas dentro de una vivienda, permitiendo su futura personalización.

Por medio de líneas horizontales se forma un sistema de zonas y márgenes Ilamada "Sistema de distribución de zonas", (Figura 01.a), estas ayudarán posteriormente a formular los estándares de las unidades dentro del soporte, así como a distinguir las posiciones más adecuadas para cierto tipo de habitaciones. Una vez dibujadas las zonas y los márgenes, se procede a denominar cada parte (Figura 01.b): La zona adyacente a la fachada es llamada Zona Alfa y se describe como un área de uso privado adyacente a una pared exterior. El espacio interno es Ilamado Zona Beta, es un área interna de uso privado que no es adyacente a una pared exterior. Como se observa, ninguna de las zonas se toca entre sí, como resultado, entre cada una de ellas, siempre hay un margen. Estos toman las características y el nombre de ambas zonas a las que son adyacentes, por consiguiente, tenemos dos Márgenes Alfa/Beta, un Margen Alfal Delta (la fachada se encuentra aquí situada) y un Margen Alfa/Gamma. La Zona Delta es un espacio exterior que en realidad es parte de la unidad de vivienda, en la mayoría de los casos es usado para balcones o porches. Al otro lado, se encuentra la Zona Gamma que puede ser interior o exterior, es de uso público y proporciona acceso a la vivienda.

La distribución de las zonas y los espacios dentro de una casa, se dan al estudiar el metraje y la función de este, lo que da como resultado un programa determinado dentro de una vivienda. Se debe de tener en cuenta que siempre existe una relación entre la función y el tamaño de un área, de acuerdo con esto, los espacios pueden ser divididos en tres grupos: Los espacios para usos especiales, que son los pensados para ser ocupado durante ciertos periodos de tiempo y que normalmen- te son nombrados según su función (dormitorio, cocina, estudio); Los espacios de usos generales, que en su mayoría, son los que tienen el mayor espacio dentro de una vivienda y pueden tener una gran variedad de combinaciones para acomodar diferentes tipos de actividades, que se espera tomen lugar simultáneamente; y por último, los espacios de servicio que se destinan a cortas ocupaciones y son de carácter utilitario (habitaciones de almacén o baños).

Para conocer las mínimas dimensiones de un espacio, debemos de analizar las actividades y el mobiliario que se utilizarán. Para ello, Habraken utiliza la tabla de estudio de espacios (Figura 02), en la que propone diferentes alternativas de acomodo del mobiliario, en habitaciones que van de los $3.24 \mathrm{~m}$ a los $20.25 \mathrm{~m}$ de profundidad y anchura. De acuerdo a esta tabla, se pueden conocer los mínimos generables para diversos espacios y plasmar dentro del soporte y con las dimensiones correctas, diversos acomodos.

Dentro de todo soporte podemos encontrar tres sectores: dos sectores alfa y un sector beta. Un sector es una zona junto con sus márgenes adyacentes. (Figura 01.c). Para comprender las distribuciones que se pueden realizar dentro de un sector se hace un análisis de

Figura 02: Tabla de estudio de espacios de J. Habraken

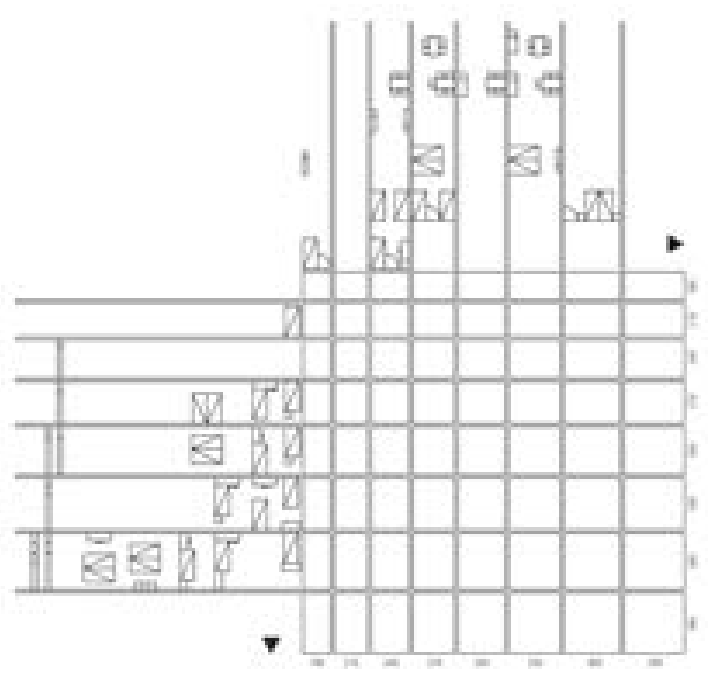

Fuente: Elaboración propia. 
los posibles usos y divisiones de este. (Figura 01.d). Los sectores pueden ser combinados de muchas maneras, incluso formar grupos interconectados. (Figura 01.e). Un grupo de sectores puede llegar a ofrecer diversas alternativas de distribución útil, pero para poder determinar las que reflejan una diferencia significativa, es necesario escoger las que representen un programa de vivienda completo, es decir, una variante básica. (Figura 01.f). El analizar un sector nos puede proporcionar datos acerca de las posibles posiciones de espacios, unidades separables, y distribuciones críticas de los espacios que tienen que ser situados en el sector.

\section{"Metodología general para la determina- ción de tipologías racionales de vivienda" de Alexander Klein}

Klein expone en su libro "Vivienda mínima" el proceso mediante el cual puede medirse la capacidad de utilización práctica de una planta antes de su ejecución. Esta metodología expone todas las condicionantes de un proyecto, reúne cierto número de ejemplos análogos resaltando sus características de manera individual, para después analizarlos, compararlos y calificarlos, lo que ayuda a eliminar variantes hasta quedarse con la mejor opción.

Dentro de su metodología existe una etapa en la que se califican de manera gráfica conceptos generales que juegan directamente con la imagen espacial: claridad, economía, forma espacial, distribución, zonas de paso, utilización de la superficie e impresión general. Algunos de ellos se han utilizado para elaborar la metodología de análisis propuesta en este artículo.

Klein analiza la ordenación de las zonas de paso y el recorrido de circulaciones, relacionando la posibilidad de organización racional y la sencillez de uso de la vivienda con el gasto de energía física, ya que según él se debe evitar la pérdida de superficie por agrandar

Figura 03: Ejemplo insatisfactorio vs ejemplo satisfactorio según Klein al estudiar recorridos, usos de superficies y zonificación.
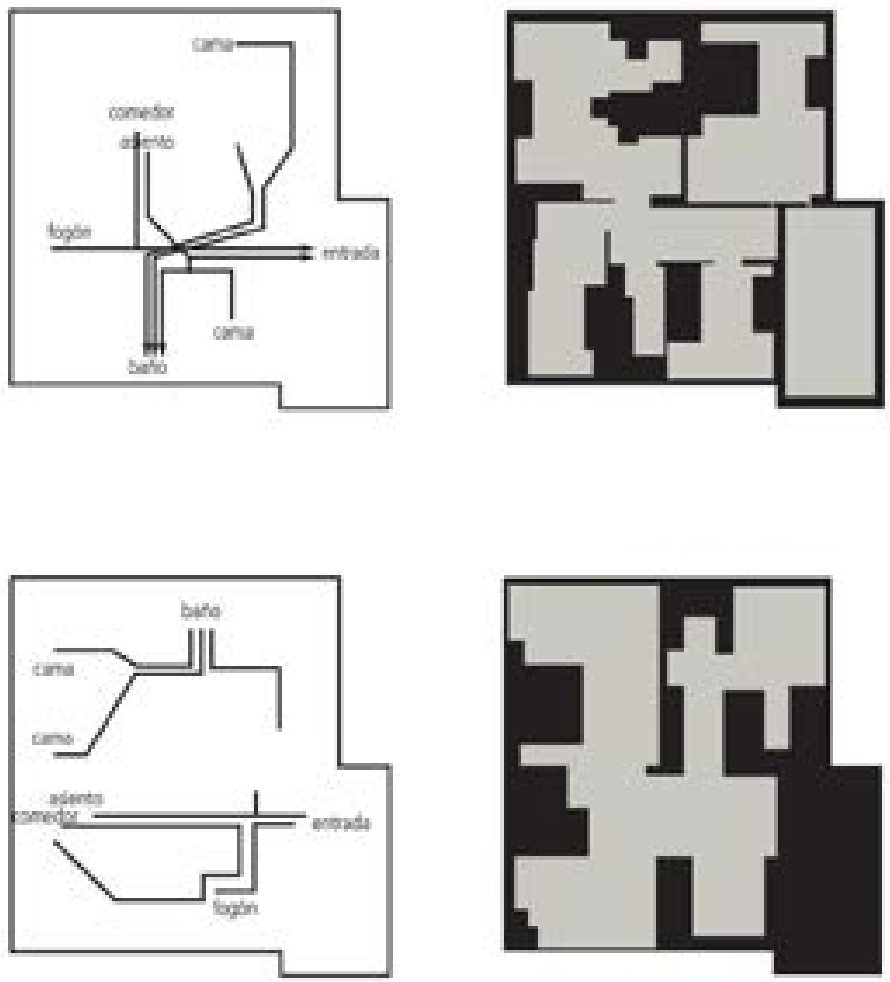
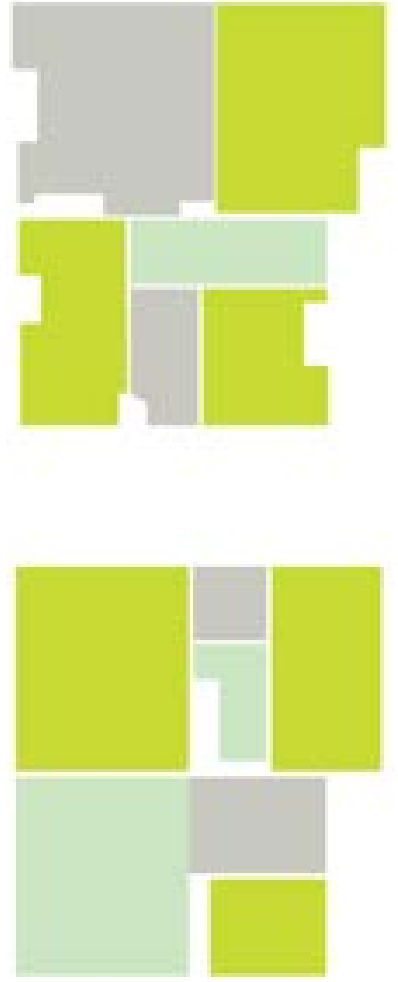

Fuente: Elaboración propia. 
Figura 04: Ejemplo de proyección de sombras con mobiliario alto y con mobiliario bajo según A. Klein.
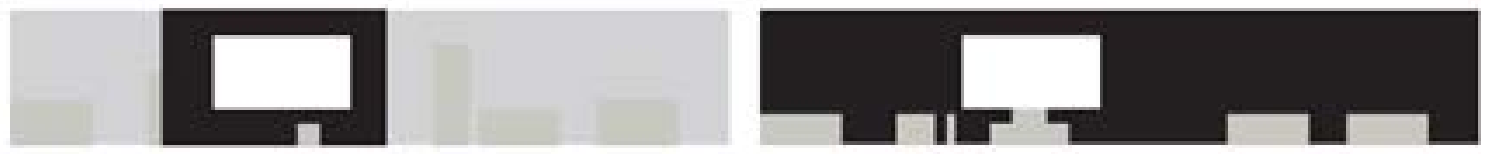

Fuente: Elaboración propia.

las zonas de circulación y los recorridos deben de ser sencillos y sin interrupciones. (Figura 03).

El alemán observa las superficies libres, aquellas que permanecen vacías tras la colocación de mobiliario imprescindible, y concluye que se debe tratar de simplificar los bordes sin dejarlos recortados, (Figura 03), para lo que es importante contar con la planta amueblada.

Posteriormente zonifica las áreas principales y se estudia la relación entre los elementos de la planta, todas aquellas superficies o zonas que, supuestas a la altura de la vista, pueden ser percibidas de un modo unitario y de las cuales depende la impresión general que el usuario tiene de la vivienda. Al igual que las superficies libres, las zonas no deben de estar fraccionadas, buscando tener menos unidades espaciales. (Figura 03).

Por último, se comparan los fraccionamientos de las superficies de pared y estrechamiento del espacio, para lo que el autor señala que aquellos muebles adosados a las paredes que superan la mitad de la altura de la habitación o que en cualquier caso superan la

Figura 05: Esquema de actividades en la vivienda según Kiyoyuki Nishihara

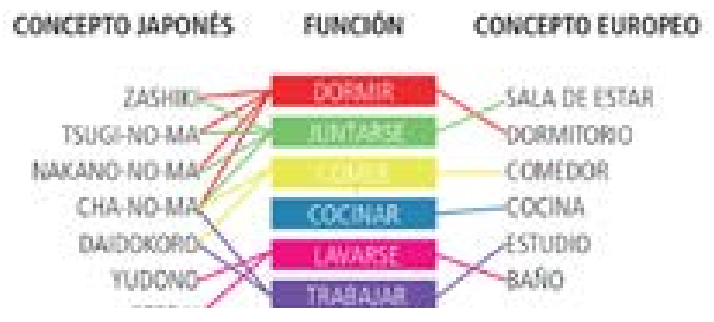

Fuente: Elaboración propia. altura de la vista, ayudan a la formación de sombras que pueden afectar a observar el espacio menos amplio, mientras que el uso de muebles bajos preserva una impresión espacial unitaria. (Figura 04).

\section{Kiyoyuki Nishihara, las actividades principa- les que se llevan a cabo en una vivienda}

Para tener una mejor comprensión de lo que es la polivalencia, es preciso conocer las actividades que una vivienda tiene que acomodar. Aunque en principio podemos identificar cientos de ellas, según Bernard Leupen, se pueden agrupar sin importar lo que represente una vivienda alrededor del mundo de acuerdo con sus similitudes. (Leupen, 2006).

En el libro "Japanese Houses: Patterns for living" Nishihara compara las casas europeas tradicionales con las casas japonesas. En Europa, explica, los espacios de las viviendas están nombrados de acuerdo con su uso (dormitorio, estudio, cocina, etc.), mientras que en Japón los espacios obtienen sus nombres de acuerdo a la relación entre unos y otros (cuarto principal, cuarto medio, el cuarto después del principal, etc.). A partir de esto, Nishihara utiliza un esquema comparativo para relacionar cada habitación con los usos que usualmente se le dan, (Figura 05), partiendo del cual, podemos concluir que las actividades principales de la vivienda se agrupan en seis: (J) juntarse, (D) dormir, (C) cocinar, (C) comer, (B) lavarse y $(\mathrm{T})$ trabajar.

Para la metodología propuesta se utilizarán para clasificar las actividades a desarrollar en cada vivienda las mencionadas por Nishihara relacionando en todos los dibujos cada actividad con los colores mostrados en la Figura 05. 


\section{El esquema de actividades básicas en la vi- vienda según Bernard Leupen}

Identificadas las actividades a desarrollar en una vivienda mediante las teorías de Nishihara, se tomó el artículo "Polyvalence, a concept for the sustainable dwelling" de Bernard Leupen en el que indica que la polivalencia no sólo depende de la organización espacial si no también de la conexión de las actividades y los lugares. Para comprobar esto Leupen esquematizó dos tipos de diagramas a través del análisis de la planta: uno que representa el sistema espacial de la vivienda (Figura 06 a), y otro que muestra cómo el usuario puede utilizar los espacios (Figura 06 b). (Leupen, 2006). Las letras que se aprecian en cada círculo de los esquemas hacen referencia a las seis actividades principales de Nishihara descritas anteriormente: (J) juntarse, (D) dormir, (C) cocinar, (C) comer, (B) lavarse y (T) trabajar. (Figura 06).

Este tipo de esquemas permiten determinar todas las variaciones fundamentales en distribuciones de espacios para apreciar la viabilidad de una estructura, "pero para tomar las decisiones lógicas, lo primero que necesitamos ver es la organización del espacio y el número de patrones y divisiones en las actividades que forman representaciones de un modo de vida". (Leupen, 2006) esto facilita por un rato el que se ordenen y se selecciones las variantes que ayuden a agrupar de mejor manera las actividades que se llevan a cabo. (Figura 06 c) y no solo nos permiten conocer la polivalencia que se le puede dar a un espacio, sino también la posible alterabilidad y extensibilidad de este, con lo que se logran sistemas de organización flexibles al poder ver el panorama completo al planificar una vivienda.

Cabe observar que en ninguna de las teorías estudiadas se toman en cuenta características como la altura de techos, color, acabados, mobiliario o iluminación, que son aspectos característicos del diseño de interiores y por tanto de la conformación de un espacio doméstico. Es por ello que en la metodología propuesta se incorporarán estos criterios en una segunda tabla de análisis comparativa que permita caracterizar estos aspectos.

\section{Descripción de la metodología de análisis del espacio doméstico polivalente}

Una vez revisados los métodos de análisis de los autores mencionados y comprendidos sus sistemas de análisis gráfico se optó por extraer los criterios de evaluación más adecuados de cada autor para entender el espacio polivalente doméstico. Tomando de Alexander Klein la forma de expresar gráficamente el uso de superficies útiles versus libres, los recorridos, la iluminación y las sombras proyectadas; de Kiyoyuki Nishihara la manera de expresar las actividades principales que se llevan a cabo en una vivienda asociadas con su metraje y su ubicación; y de Bernard Leupen sus formas de

Figura 06: Esquema de relación entre los espacios de una vivienda, esquema de relación entre las actividades y el espacio de la misma vivienda y variación del esquema de relación entre las actividades y el espacio de la misma según Bernard Leupen.
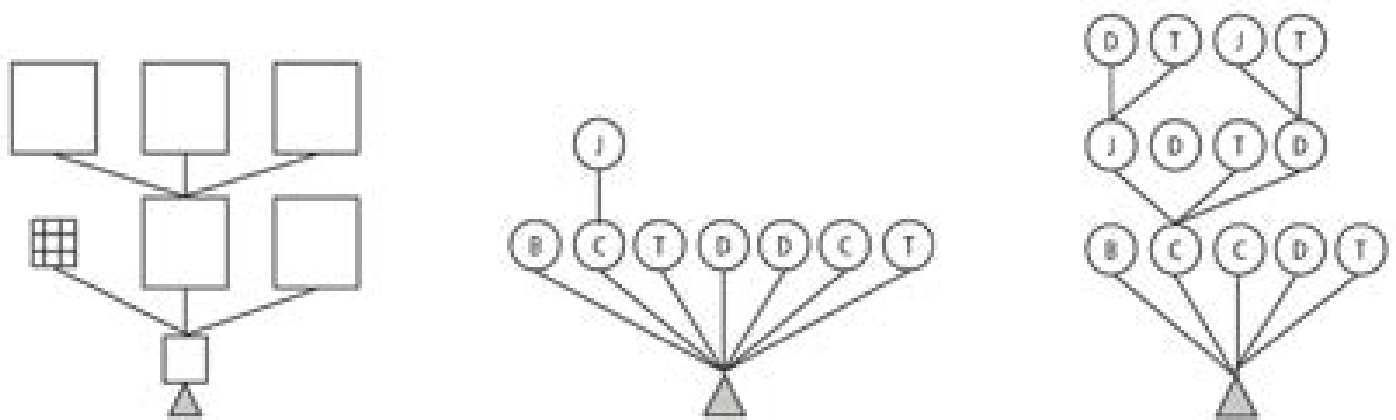

Fuente: Elaboración propia. 
esquematización de la relación de los espacios y las actividades de la vivienda. Complementando todo esto con las plantas y los cortes con los movimientos del mobiliario representados por códigos de color y tipo de línea explicados en este apartado del texto, la iconografía que indica los tipos de movimientos existentes en los elementos de cada proyecto y la superficie del proyecto.

Para establecer este método comparativo del espacio polivalente se diseñó una ficha de análisis tipo que recogiera todos los criterios anteriormente citados. (Figura 07). Como se puede observar en esta, la información se encuentra distribuida en dos partes: La primera está conformada por la información principal del proyecto, dividida en la barra de título, el resumen del proyecto, las imágenes, los materiales generales, la planta, el corte y, por último, como resultado del análisis general, la iconografía. Mientras que la segunda parte, se conforma por ocho esquemas de análisis: comparación de superficies entre la planta del proyecto y las dimensiones mínimas de vivienda en el Distrito Federal, la superficie útil de la planta versus la superficie libre, las distribución de las zonas de acuerdo a las principales actividades que se llevan a cabo en una vivienda, los esquemas de Leupen en cuanto a relación de espacios y actividades, los recorridos principales, la entrada de luz natural en el espacio y la proyección de sombras.

De manera más específica y detallada, los elementos contenidos en esta ficha de análisis son los siguientes:

Barra de título contiene los elementos esenciales para poder identificar cada caso de estudio (Figura 08): el número de caso a analizar (a), el nombre del proyecto (b), el arquitecto, diseñador o despacho responsable (c) y el año en el que fue creado (d).

Resumen del proyecto (e), escrito por el propio diseñador o arquitecto en el que brevemente se explica el propósito de su creación. (Figura 08).

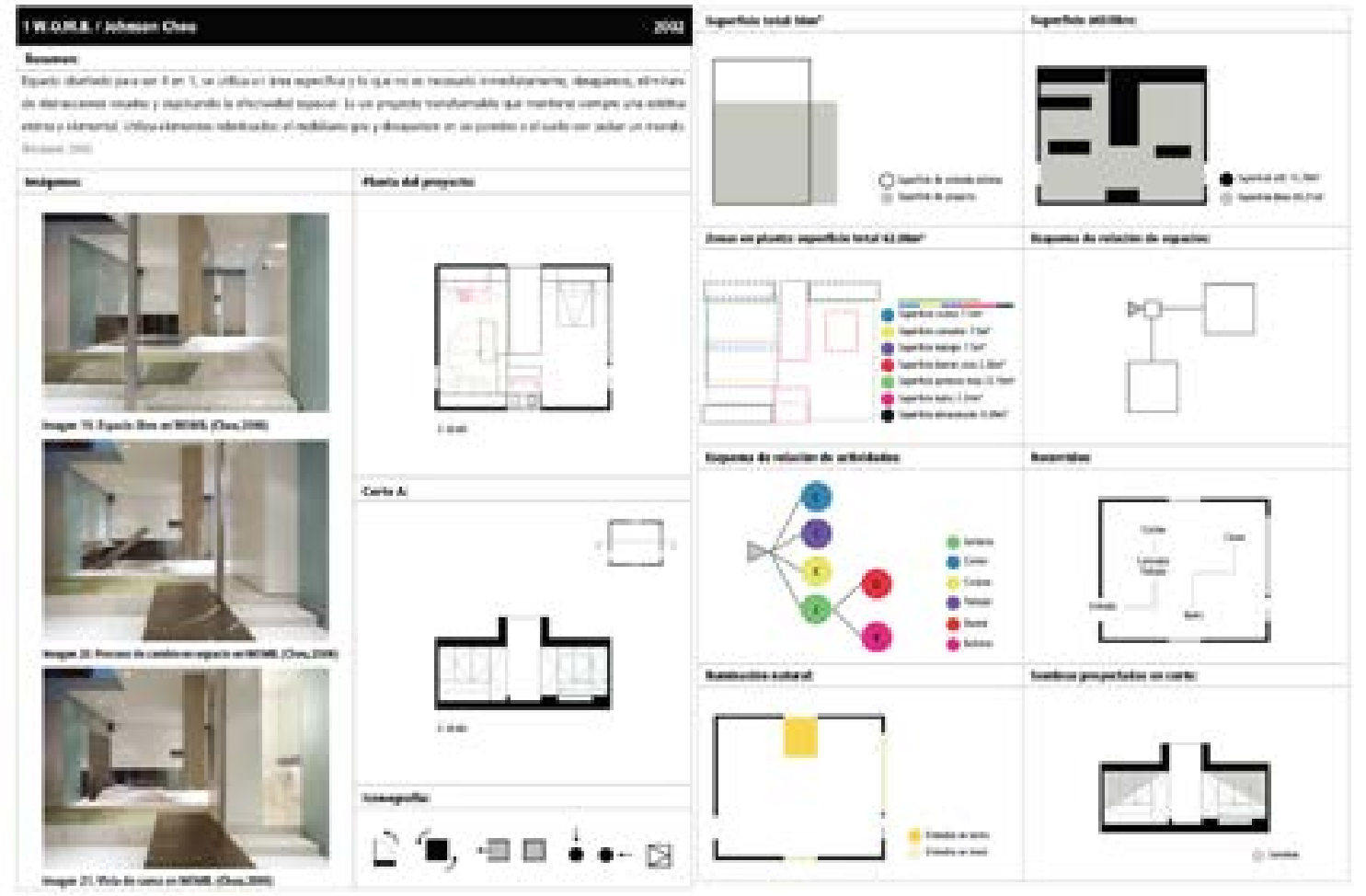

Fuente: Elaboración propia. 
Perspectivas, imágenes y fotografías interiores del proyecto ( $f$ ), las cuales muestran el movimiento de elementos que lo componen, los materiales utilizados, las entradas de luz natural e incluso la interacción con personas, lo cual nos ayuda a conocer la escala del lugar y el uso de diversos componentes que lo conforman. (Figura 09).

Planta (g) y corte (h) siempre representados a la misma escala que ayudan a comprender el espacio de manera más real. Además de mostrar la distribución interior, las plantas y los cortes también ayudan a identificar, caracterizar y clasificar los movimientos de las particiones y el mobiliario. (Figura 09). Esto es posible, gracias al código de colores y tipos de líneas mostrados en la tabla de descripción de movimientos. (Figura 10). Estos códigos facilitan el entendimiento de la articulación de los elementos del proyecto entre sí y con el espacio y permiten identificar cuál es el movimiento más adecuado para cada tipo de actividad y de espacio con el fin de conseguir una mayor polivalencia. En la tabla, se especifica el tipo de movimiento que puede realizar un elemento, en esta también se puede encontrar una explicación gráfica en planta, corte y axonometría del proceso por el cual un objeto que sufre este movimiento cambia de ubicación.

Figura 08: Detalle de encabezado de la ficha de análisis.

a.

Rename:

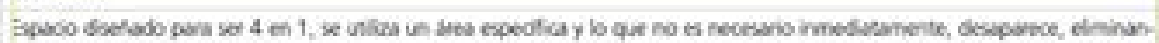

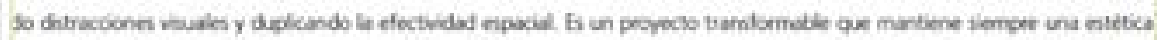

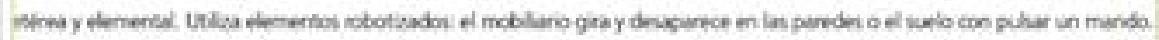
Mecies moe

Fuente: Elaboración propia.

Fiaura 09: Detalle de perspectivas. planta corte e iconos de la ficha de análisis

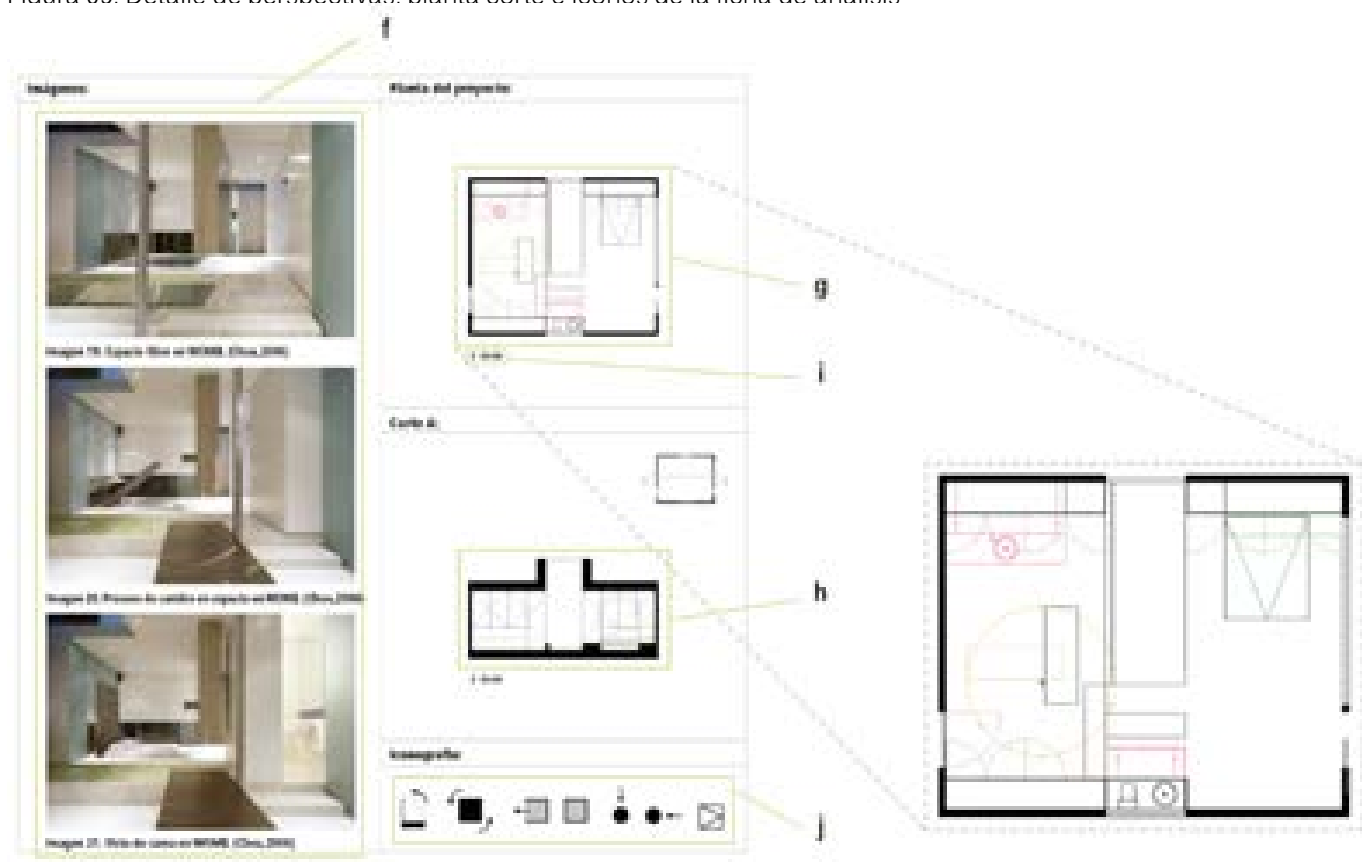

Fuente: Elaboración propia. 
Se anexa igualmente un ícono que identifica cada movimiento y que se utilizará como representación del tipo de movimiento. Por otro lado, y asociados a cada movimiento, también se incluyen un código de color y tipo de línea utilizados en planta y en corte.

Materiales y acabados ( $k$ ) se reconocen y hacen explícitos a través de las imágenes y se han clasificado en dos tipos: acabados de interior (piso, paredes y techo) y acabados de mobiliario.

Iconos (j), ya mencionados para la tabla de descripción de movimientos, ilustran el tipo de movimiento, mobiliario, iluminación y almacenamiento. Se representan en color gris, todos los movimientos existentes, y se resaltan de entre estos en negro los que corresponden al proyecto. (Figura 09).

Diagrama de superficie total de la vivienda expresada en $\mathrm{m} 2(\mathrm{~m})$ comparada con la superficie de vivienda mínima en Ciudad de México estipulada por el INFONAVIT (62 m2). (n). (Figura 11).

Esquema de relación entre la superficie útil y la superficie libre (o), en el cual se representa en color negro las zonas ocupadas por estructuras o mobiliario y en color gris el espacio que queda libre, complementado con el metraje

Figura 10: Tabla de descripción de movimientos

\begin{tabular}{|c|c|c|c|c|c|}
\hline $\begin{array}{l}\text { Tipo de } \\
\text { movimiento }\end{array}$ & Perspectiva & $\begin{array}{l}\text { Representación } \\
\text { en planta }\end{array}$ & $\begin{array}{l}\text { Aleppresentadón } \\
\text { en corte }\end{array}$ & Ícono & $\begin{array}{l}\text { Tipo de } \\
\text { linea }\end{array}$ \\
\hline $\begin{array}{c}\text { Sin } \\
\text { movimiento }\end{array}$ & & & & & ............. \\
\hline Lateral & & & - & -1 & 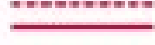 \\
\hline Gratorio & & & & & …… \\
\hline $\begin{array}{c}\text { Puerta } \\
\text { apertura } \\
\text { herizantal }\end{array}$ & & & & & \\
\hline $\begin{array}{l}\text { Puerta } \\
\text { apertura } \\
\text { vertical }\end{array}$ & & & & & $\ldots . . .$. \\
\hline Persiana & & $\infty$ & $\square$ & & nennen \\
\hline Corrediza & & & & & $\ldots$ \\
\hline Biombo & & & 44 & & nan. \\
\hline Escalera & & & $E$ & & 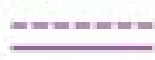 \\
\hline
\end{tabular}

Fuente: Elaboración propia. 
de cada una de las superficies. Este esquema sirve para, en caso de ser necesario mejorar la habitabilidad, agrupar y dejar los bordes lo más simple posible. (Figura 11).

Diagrama de las zonas en planta, en el que zonifica cada espacio de la vivienda representando cada zona con un color sólido. A diferencia del análisis de Klein, en este caso se analizarán espacios polivalentes que albergan más de una actividad de manera simultánea, por lo que la zonificación se realizará a base de recuadros punteados ( $p$ ), que ayudan a observar de manera ordenada la superposición de áreas. De igual manera este mismo fenómeno se representa en las barras de color del lado derecho (q), señalando que zonas ocupan el mismo lugar que otras. Como apoyo al diagrama, se expresa la dimensión de cada una de las zonas en m2 (r), y se suman para darnos la superficie total de acuerdo a la zonificación de la vivienda (s). Este dato nos refleja la efectividad del uso del espacio, es decir, cuanto mayor sea el número versus el metraje de la superficie total anteriormente señalada, mejor estará calificado en la escala de polivalencia. (Figura 12).

Esquema de relación de espacios. Este esquema ayuda a identificar las áreas que están aisladas o separadas por medio de estructura interna o mobiliario gracias a que a través de recuadros se representan los espacios y que con el uso de líneas se indican sus conexiones y cómo acceder a ellos. (Figura 12).

Esquema de relación de actividades, que permite ordenar y agrupar de mejor manera las actividades que se llevan a cabo de acuerdo a las zonas de la vivienda. Las letras que se aprecian en cada círculo hacen referencia a las seis actividades principales descritas anteriormente por Nishihara: (J) juntarse, (D) dormir, (C) cocinar, (C) comer, (B) lavarse y (T) trabajar. (Figura 12).

Diagrama de planificación de los recorridos dentro de la vivienda. (u). Este muestra la complejidad del uso del esquema de actividades que el diseñador propone. Usando el mismo

Figura 11: Detalle de superficie total y superficie útil/libre de la ficha de análisis
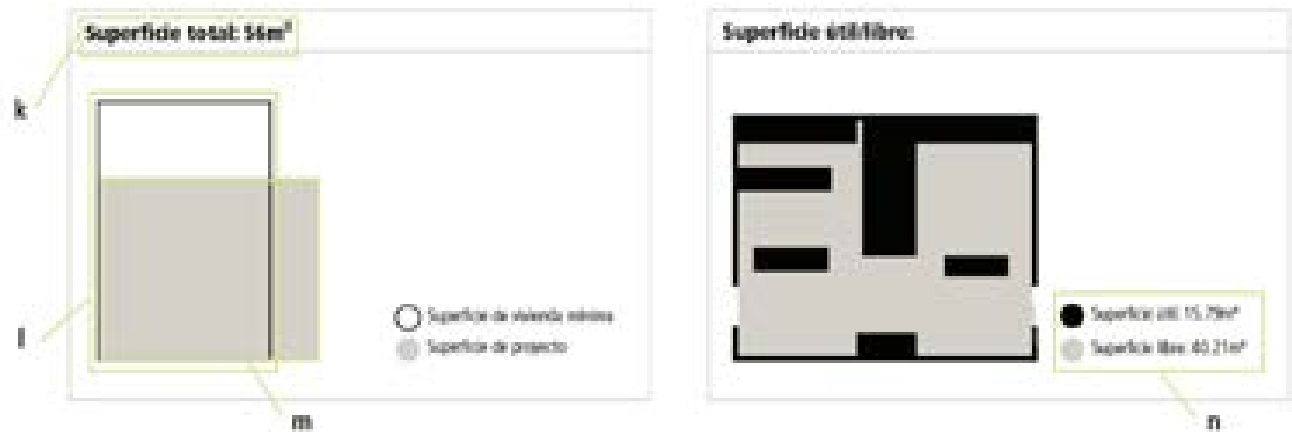

Fuente: Elaboración propia.

Fiaura 12: Detalle de zonas en planta. relación de espacios v relación de actividades de la ficha de análisis.

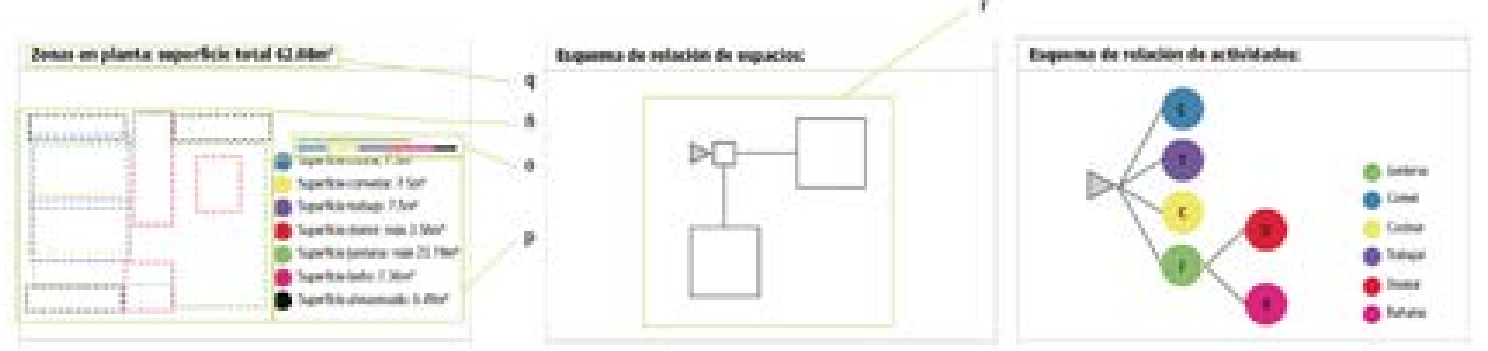

Fuente: Elaboración propia. 
esquema de Klein, se dibujan cuatro recorridos principales: entrada - comedor, entrada trabajo, cocina - comedor y cama - baño. Entre más corto y simple sea el recorrido, mejor se considera el uso del lugar.

Esquema de iluminación natural. Considerado bajo dos categorías: las entradas por el techo (v) y las entradas por los muros (w). Estas entradas se encuentran señaladas por recuadros de color en la planta de cada proyecto estudiado.

Esquema de sombras proyectadas. Gracias a este se puede conocer la ubicación de las principales entradas de luz natural y se señalan los lugares donde sería necesario introducir luz artificial para que todo el espacio se encontrara igualmente iluminado.

Esta ficha de análisis se puede aplicar en cada uno de los casos que se requiera analizar. El uso de dibujos con un código gráfico idéntico para todos los casos, una escala gráfica constante y el mapeo consecutivo de todos los puntos descritos anteriormente con un criterio uniforme permite la comparación sencilla de distintas variables entre cada uno de los ejemplos que se estudien. Este proceso de comparación de casos de estudio se considera necesario para poder extraer conclusiones que sirvan para elaborar estrategias de diseño que faciliten la toma de decisiones a la hora de querer diseñar un espacio doméstico polivalente. La comparación se puede realizar directamente desde la ficha analítica, aunque se considera más práctica la comparación de los casos por variables específicas. De esta manera se aíslan estas y se pueden detectar más fácilmente relaciones entre los distintos ejemplos. Este es el caso de la Figura 14, en el que se resume la información de diez casos de estudio por columna y se comparan por fila las variables de forma del espacio, movilidad en la configuración de espacios interiores, tipo de mobiliario, entradas de luz, materiales utilizados de manera general y el tipo de almacenamiento, pudiendo extraer conclusiones más fácilmente.

\section{Resultados y conclusiones:}

La metodología diseñada se implementó para el estudio de diez casos de espacios domésticos polivalentes contemporáneos de distintas localizaciones y diseñadores seleccionadas para recopilar la mayor diversidad de modos de alcanzar la polivalencia a través del mobiliario y el mayor uso de distintos materiales: WOMB de Johnson Chou (2002), Circuit Box de Studio X Design Group (2004), Insider de ELII (2011), Bath Kitchen House de Takeshi Shikauchi (2012), Barcode Room de Studio_01 (2012), Paris Apartment de Kitoko Studio (2014), Berlin Apartment de Sparoom + John Paulcoss (2015), Biombastic de ELII (2015), Riviera Cabin de Ilab (2017) y Yojigen Poketo de ELII (2017). Se volcó la información de cada proyecto en la ficha diseñada y se compararon según la tabla comparativa de casos de estudio extrayendo los siguientes resultados y conclusiones:

En cuanto a la forma de las plantas, en su mayoría son regulares, es decir, cuadrados o rectángulos. Sus líneas son rectas, a excepción de dos proyectos que tienen bordes asimétricos. Este tipo de formas propician una

Figura 13: Detalle de recorridos, iluminación natural y sombras proyectadas en corte de la ficha de análisis.
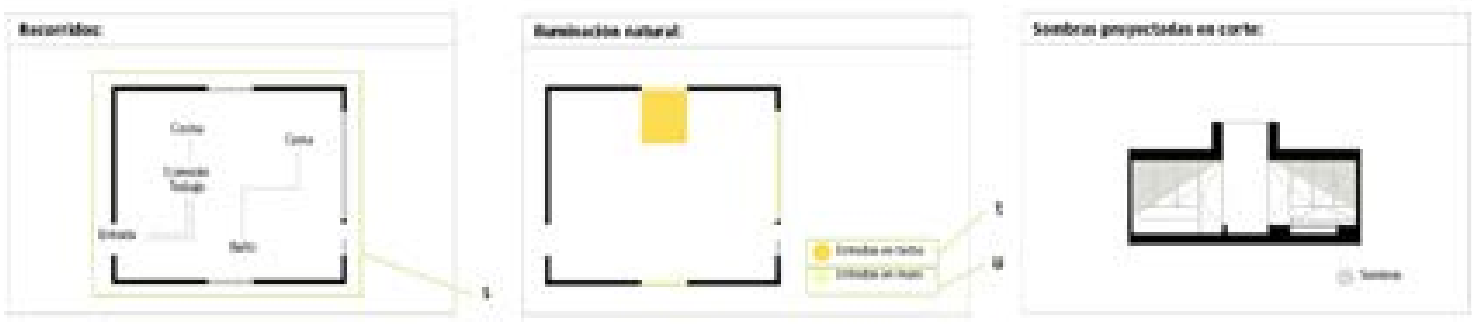

Fuente: Elaboración propia. 
organización el espacio más eficiente, agrupando los muebles, los módulos de servicio y de almacenamiento en el perímetro, para así poder concentrar el espacio libre con una forma regular y en el centro.

Una característica destacable de todos los proyectos es que las actividades pueden tomar lugar en más de una zona, gracias a elementos móviles como paredes, biombos y mobiliario que ayudan a ocupar o liberar el espacio, de acuerdo a la actividad a desarrollar. Estos elementos se encuentran fijados a paredes, techos o pisos y se despliegan a base de carriles, ruedas y bisagras.

En todos los proyectos se observa el uso de puertas correderas horizontales junto a elementos que se mueven de manera lateral. Mientras que los elementos tipo persiana o biombo se encuentran en la minoría de los casos.

En la mayoría de los ejemplos se utilizan materiales de colores claros que propician la reflexión de la luz. Los muebles se encuentran ocultos en paredes o en piso, varios de los cuales, son transformables, es decir, se utilizan para más de un propósito, lo que ocasiona que se reduzca el número de objetos dentro de la vivienda, lo que potencia la sensación de amplitud espacial.
Los materiales más utilizados son el laminado de color gris y la madera de tono claro para pisos, paredes y techos pintados o revestidos de color blanco, así como metal y madera (natural o pintada) para la fabricación de mobiliario, materiales baratos, ligeros, resistentes y versátiles.

En lo referido al mobiliario, se observa que este debe de permanecer oculto, porque ayuda a generar un espacio más amplio, aspecto que se busca a toda costa para evitar la sensación de tensión espacial considerando que el espacio es reducido.

Otro aspecto importante que se ha identificado, es use los proyectos tratan de evitar los cambios de nivel de piso para permitir que el espacio sea. Los únicos desniveles que se encuentran son los que marcan un cambio de zona y son aprovechados como espacio de almacenamiento ya que es imprescindible aprovechar cada zona vacía para agregar espacio de guardado.

En cuanto a los usos y actividades, se observa que la zona de estancia (J) siempre es la más extensa, y es en la que además, por medio de mobiliario especial, se desarrollan las actividades de comer y trabajar, cuya ubicación puede variar y modificarse por el usuario. Por otro lado, las zonas de baño y cocina, son permanentes

Figura 14: Extracto de la tabla comparativa de casos de estudio por variable

\begin{tabular}{|c|c|c|c|c|c|c|c|c|c|c|}
\hline $\cos x$ & 1 & 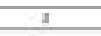 & 1 & + & 1 & 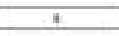 & 1 &. & 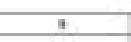 & 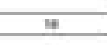 \\
\hline$=$ & & Dat & & & & W & 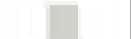 & $=$ & & \\
\hline istis. & $\begin{array}{l}\mathrm{LI}^{2}= \\
-\mathbf{n}-\mathbf{n}\end{array}$ & $\begin{array}{c}-1- \\
81\end{array}$ & $\begin{array}{c}\mathbf{E} \boldsymbol{1}, 7 \\
-\mathbf{n}\end{array}$ & EI, & $-\frac{1}{T_{j}}$ & $\begin{array}{c}-\mathbf{a}-\boldsymbol{T} \\
=\end{array}$ & $15,-$ & $\mathrm{r}, \mathrm{L}-2$ & E & $\begin{array}{r}-\mathbf{a} / 1 \\
\Gamma, 2\end{array}$ \\
\hline nown & 르 ㅁ & 리 & 피 & $\square$ & - $]$ & 피 & 口 & 르 & 듬 & 미 \\
\hline insin & $\bullet \bullet-$ & •- & $\bullet-$ & - - & $\bullet \bullet-$ & $\bullet-$ & $\bullet-$ & $\bullet-$ & $\bullet-$ & $\bullet-$ \\
\hline ansunit & $=$ & $=$ & - & - & - & - & -1 & $-\infty$ & - & - \\
\hline$m$ & $\nabla$ & $\triangle$ & $\nabla$ & 口曰⿴囗十 & $\square$ & $\square \mathbb{B}$ & $\triangle \square$ & $\otimes$ & $\square \otimes \square$ & 죠 \\
\hline
\end{tabular}

Fuente: Elaboración propia. 
y no cambian su ubicación, ya que deben de tener acceso directo a instalaciones como agua, drenaje y gas.

Sobre los recorridos, se observa que son simples. Se trata en la mayoría de los casos de tener el dormitorio y el baño cerca, al igual que el comedor y la cocina. De este modo, no se gastan energías físicas innecesarias y las actividades pueden desempeñarse de manera más ordenada. Además, se busca no tener elementos que obstruyan el paso y hagan el recorrido más tardado.

En varios casos se localizan balcones o grandes ventanas que ayudan a abrir el espacio permitiendo la entrada de luz y ventilación, mientras que sólo en dos casos se observa la entrada de luz por el techo.

Tras las conclusiones del análisis de los diez espacios domésticos polivalentes seleccionados se identifica la "metodología de análisis del espacio doméstico polivalente" como una herramienta para extraer estrategias de diseño que hagan el espacio doméstico más eficiente. Este es un recurso destacable a la hora de diseñar viviendas con una superficie mínima en la que se quieran desarrollar el mayor número de actividades posibles de manera simultánea o traslapada. Igualmente se considera interesante a la hora de diseña un espacio doméstico aplicar esta metodología de análisis a las diversas variantes que se vayan generando para así poder descartar unas $u$ otras de una manera más razonada atendiendo a principios de optimización, simultaneidad, flexibilidad y eficiencia.

Por último, se considera el método de análisis propuesto como punto de inicio de futuras investigaciones. Entre estas se encuentra el desarrollo de una aplicación informática que permita dar pautas de diseño a nuevos espacios polivalentes y evaluar y verificar los datos de estos a partir de los datos proporcionados por esta forma de análisis del espacio doméstico polivalente.

\section{Bibliografía:}

Carboni, I. (2015). La flexibilidad en la vivienda colectiva contemporánea. Propuesta de seis modelos tipológicos. (Tesis maestría inédita). Universidad Politécnica de Cataluña. Escuela Técnica Superior de Arquitectura de Barcelona, Barcelona. Recuperado de: https://issuu.com/icarboni/docs/la_flexibilidad_en_la_vivienda_cole

Habraken, N. J. (1979). El diseño de Soportes. Barcelona: Editorial Gustavo Gili, SA.

Hertzberger, H. (2016). Diagoon Housing Delft. 1967-1970. Delft: Herman Hertzberger. Recuperado de: ttps://www. hertzberger.nl/images/nieuws/DiagoonHousingDelft2016.pdf

INFONAVIT. (2018). Cumbre INFONAVIT 2018. México: INFONAVIT.

INFONAVIT. (2006). Disposiciones y reglamentos de vivienda-2006. México: INFONAVIT. Recuperado de: http://studylib.es/doc/5605343/disposiciones-y-reglamentos-de-vivienda-2006

Klein, A. (1980). Vivienda mínima: 1906-1957. Barcelona: Gustavo Gili, SA.

Leupen, B. (2006). Polyvalence, a concept for the sustainable dwelling. Nordic Journal of Architectural Research, V. 19, No 3, pp. 23-31. Recuperado de: https://www.google.com/url?sa=t\&rct=i\&q=\&esrc=s\&source=web\&cd=1\&ved=2ahUKEwj67abRrJjdAhWTw4MKHQL-BycQFjAAegQIARAC\&url=http\%3A\%2F\%2Farkitekturforskning.net\%2Fna\%2Farticle\%2Fdownload\%2F156\%2F120\&usg=AOvVaw0Jcx-xrujy1eTQys2xqkeV

Leupen, B., \& Mooij, H. (2011). Housing Design. Rotterdam: NAi Publishers.

Nishihara, K. (1971). Japanese houses: Patterns for living. Japón: Japan Publications Inc.

Sánchez, S. I. (2004). El espacio doméstico en Buenos Aires: concepciones, modelos e imaginarios (1872-1935). Buenos Aires: Facultad de Filosofía y Letras Universidad de Buenos Aires. (Tesis Doctoral). 